\title{
ON THE STABILITY OF DELAY INTEGRO-DIFFERENTIAL EQUATIONS
}

\author{
Ali Fuat Yeniçerioğlu* and Salih Yalçınbaş** \\ *Department of Mathematics, Faculty of Education, Kocaeli University, 41380 \\ Kocaeli, Turkey. fuatyenicerioglu@hotmail.com \\ **Department of Mathematics, Faculty of Science, Celal Bayar University, 45040 \\ Manisa, Turkey. salih.yalcinbas@bayar.edu.tr
}

\begin{abstract}
Some new stability results are given for a delay integro-differential equation. A basis theorem on the behavior of solutions of delay integro-differential equations is established. As a consequence of this theorem, a stability criterion is obtained.
\end{abstract}

Keywords - Integro-differential equation, Stability, Delay.

\section{INTRODUCTION AND PRELIMINARIES}

This paper deals with the stability of the trivial solution for a delay integrodifferential equation. An estimate of the solutions is established and sufficient conditions for the stability and the asymptotic stability of the trivial solution are given. Since the first systematic study was carried out by Volterra [10], this type of equations have been investigated in various fields, such as mathematical biology and control theory (see, e.g., $[4,5,8])$.

In this paper, we will give a basic theorem on the behavior of solutions of scaler delay integro-differential equations. As a consequence of this theorem, we will establish a criterion for the stability of the trivial solution; see [3,6,7,9] for related stability results. A root of the associated characteristic equation is used in obtaining our results. For the basis theory of integral equations, we choose to refer to the books by Burton [1] and Corduneanu [2].

Note that linear neutral delay differential equations with periodic coefficients have been studied by Philos and Purnaras [9] and the behavior of solutions of linear differential equations with unbounded delay have been obtained by Kordonis and Philos $[6]$.

Let us consider initial value problems for delay integro-differential equations with a delay $\tau>0$,

$$
\begin{array}{crl}
x^{\prime}(t)= & a x(t)+b x(t-\tau)+c \int_{t-\tau}^{t} x(s) d s, & t \geq 0 \\
x(t)=\phi(t), & -\tau \leq t \leq 0
\end{array}
$$

where $a, b$ and $c$ are real number, $\phi(t)$ is continuous given initial function on the segment $[-\tau, 0]$.

By a "solution" of the delay integro-differential equation (1), we mean a continuous real-valued function $x$ defined on the real line $I R$, which is continuously differentiable on the interval $[0, \infty)$ and satisffies (1) for all $t \geq 0$. 
It is known (see, for example, [1]) that, for any given initial function $\phi$, there exists a unique solution $x$ of the delay integro-differential equation (1), where $\phi$ is defined in (2), $x$ will be called the solution of the initial problem (1)-(2) or, more briefly, the solution of (1)-(2).

If we look for a solution of (1) of the form $x(t)=e^{\lambda t}$ for $t \in I R$, we see that $\lambda$ is a root of the "characteristic equation"

$$
\lambda=a+b e^{-\lambda \tau}+c \int_{0}^{\tau} e^{-\lambda s} d s \quad \text { or } \quad \lambda=a+b e^{-\lambda \tau}+c \lambda^{-1}\left(1-e^{-\lambda \tau}\right)
$$

Now, let us introduce the hypothesis

$$
b e^{-\gamma \tau}+c \gamma^{-1}\left(1-e^{-\gamma \tau}\right)>\gamma-a \quad \text { and } \quad|b| \tau e^{-\gamma \tau}+|c| \gamma^{-1}\left[\gamma^{-1}\left(1-e^{-\gamma \tau}\right)-\tau e^{-\gamma \tau}\right] \leq 1 .
$$

Under this hypothesis, the characteristic equation (3) has a unique root $\lambda_{0}$ in the interval $(\gamma, \infty)$; this root is such that

$$
|b| \tau e^{-\lambda_{0} \tau}+|c| \lambda_{0}^{-1}\left[\lambda_{0}^{-1}\left(1-e^{-\lambda_{0} \tau}\right)-\tau e^{-\lambda_{0} \tau}\right]<1
$$

Indeed, let $F(\lambda)$ denote the characteristic function of (1), i.e.,

$$
F(\lambda)=\lambda-a-b e^{-\lambda \tau}-c \int_{0}^{\tau} e^{-\lambda s}=\lambda-a-b e^{-\lambda \tau}-c \lambda^{-1}\left(1-e^{-\lambda \tau}\right)
$$

Since $\lambda=0$ is a removable singularity of $F(\lambda)$, we can regard $F(\lambda)$ as a entire function with $F(0)=-(a+b+c \tau)$. Then from the first inequality of (4), it follows that $F(\gamma)<0$. Moreover, by the secod inequality of (4), we obtain for $\lambda>\gamma$,

$$
\begin{aligned}
F^{\prime}(\lambda) & =1+b \tau e^{-\lambda \tau}+c \int_{0}^{\tau} s e^{-\lambda s} d s=1+b \tau e^{-\lambda \tau}+c \lambda^{-1}\left[\lambda^{-1}\left(1-e^{-\lambda \tau}\right)-\tau e^{-\lambda \tau}\right] \\
& \geq 1-|b| \tau e^{-\lambda \tau}-|c| \lambda^{-1}\left[\lambda^{-1}\left(1-e^{-\lambda \tau}\right)-\tau e^{-\lambda \tau}\right] \\
& >1-|b| \tau e^{-\gamma \tau}-|c| \gamma^{-1}\left[\gamma^{-1}\left(1-e^{-\gamma \tau}\right)-\tau e^{-\gamma \tau}\right] \geq 0,
\end{aligned}
$$

and consequently, the function $F$ is strictly increasing on the interval $(\gamma, \infty)$. Furthermore, for every $\lambda \geq \gamma$, we have

$$
F(\lambda) \geq \lambda-a-|b| e^{-\lambda \tau}-|c| \lambda^{-1}\left(1-e^{-\lambda \tau}\right)
$$

and so, $F(\infty)=\infty$. Thus, in the interval $(\gamma, \infty)$, the equation $F(\lambda)=0$ has a unique solution $\lambda_{0}$. It follows from the second inequality of (4) that 
$|b| \tau e^{-\lambda_{0} \tau}+|c| \lambda_{0}^{-1}\left[\lambda_{0}^{-1}\left(1-e^{-\lambda_{0} \tau}\right)-\tau e^{-\lambda_{0} \tau}\right]<|b| \tau e^{-\gamma \tau}+|c| \gamma^{-1}\left[\gamma^{-1}\left(1-e^{-\gamma \tau}\right)-\tau e^{-\gamma \tau}\right] \leq 1$

i.e., $\lambda_{0}$ satisfies (5). Note that (5) implies in particular that

$$
1+b \tau e^{-\lambda_{0} \tau}+c \lambda_{0}^{-1}\left[\lambda_{0}^{-1}\left(1-e^{-\lambda_{0} \tau}\right)-\tau e^{-\lambda_{0} \tau}\right]>0 .
$$

Before closing this section, we will give two well-known definitions (see, for example, [1]). The trivial solution of (1) is said to be "stable" (at 0 ) if for every $\varepsilon>0$, there exists a number $\delta=\delta(\varepsilon)>0$ such that, for any initial fuction $\phi$ with

$$
\|\phi\| \equiv \max _{-\tau \leq t \leq 0}|\phi(t)|<\delta
$$

the solution $x$ of (1)-(2) satisfies

$$
|x(t)|<\varepsilon, \quad \text { for all } t \in[-\tau, \infty) \text {. }
$$

Moreover, The trivial solution of (1) is called "asymptotically stable" (at 0 ) if it is stable in the above sense and in addition there exists a number $\delta_{0}>0$ such that, for any initial fuction $\phi$ with

$\|\phi\|<\delta_{0}$, the solution $x$ of (1)-(2) satisfies

$$
\lim _{t \rightarrow \infty} x(t)=0
$$

\section{MAIN RESULTS}

The main results of the paper are the following theorem and a corollary of this theorem.

Theorem. Assume that (4) holds and let $\lambda_{0}$ be the unique root of (3) in the interval $(\gamma, \infty)$. Then the solution $x$ of (1)-(2) satisfies

$$
\begin{aligned}
& \left|e^{-\lambda_{0} t} x(t)-\frac{L(\phi)}{1+b \tau e^{-\lambda_{0} \tau}+c \lambda_{0}^{-1}\left[\lambda_{0}^{-1}\left(1-e^{-\lambda_{0} \tau}\right)-\tau e^{-\lambda_{0} \tau}\right.}\right| \\
& \leq M(\phi)\left\{|b| \tau e^{-\lambda_{0} \tau}+|c| \lambda_{0}^{-1}\left[\lambda_{0}^{-1}\left(1-e^{-\lambda_{0} \tau}\right)-\tau e^{-\lambda_{0} \tau}\right]\right\}, \quad \forall t \geq 0
\end{aligned}
$$

where 


$$
L(\phi)=\phi(0)+b e^{-\lambda_{0} \tau} \int_{-\tau}^{0} e^{-\lambda_{0} \tau} \phi(r) d r+c \int_{0}^{\tau} e^{-\lambda_{0} s}\left[\int_{-s}^{0} e^{-\lambda_{0} r} \phi(r) d r\right] d s
$$

and

$$
M(\phi)=\max _{-\tau \leq t \leq 0}\left|e^{-\lambda_{0} t} \phi(t)-\frac{L(\phi)}{1+b \tau e^{-\lambda_{0} \tau}+c \lambda_{0}^{-1}\left[\lambda_{0}^{-1}\left(1-e^{-\lambda_{0} \tau}\right)-\tau e^{-\lambda_{0} \tau}\right.}\right| .
$$

Proof. Let now $x$ be the solution of (1)-(2).

Define

$$
y(t)=e^{-\lambda_{0} t} x(t), \quad \text { for } t \in[-\tau, \infty) .
$$

Then it is easy to see that the fact that $x$ satisfies (1) for all $t \geq 0$ is equivalet to

$$
y^{\prime}(t)=\left(a-\lambda_{0}\right) y(t)+b e^{-\lambda_{0} \tau} y(t-\tau)+c \int_{0}^{\tau} e^{-\lambda_{0} s} y(t-s) d s, \quad t \geq 0
$$

Moreover, the initial condition (2) can be equivalently written

$$
y(t)=e^{-\lambda_{0} t} \phi(t), \quad \text { for } t \in[-\tau, 0] .
$$

Furthermore, by using the fact that $\lambda_{0}$ is a root of (3) and taking into account (8) and (11), we can verify that (10) is equivalent to

$$
y(t)=-b e^{-\lambda_{0} \tau} \int_{t-\tau}^{t} y(r) d r-c \int_{0}^{\tau} e^{-\lambda_{0} s}\left\{\int_{t-s}^{t} y(r) d r\right\} d s+L(\phi), \quad t \geq 0 .
$$

Next, by taking into account (6), we can define

$$
z(t)=y(t)-\frac{L(\phi)}{1+b \tau e^{-\lambda_{0} \tau}+c \lambda_{0}^{-1}\left[\lambda_{0}^{-1}\left(1-e^{-\lambda_{0} \tau}\right)-\tau e^{-\lambda_{0} \tau}\right]}, \quad t \in[-\tau, \infty) .
$$

Then we can see that (12) reduces to the following equivalent equation:

$$
z(t)=-b e^{-\lambda_{0} \tau} \int_{t-\tau}^{t} z(r) d r-c \int_{0}^{\tau} e^{-\lambda_{0} s}\left\{\int_{t-s}^{t} z(r) d r\right\} d s, \quad t \geq 0 .
$$

On the other hand, (11) takes the equivalent form

$$
z(t)=e^{-\lambda_{0} t} \phi(t)-\frac{L(\phi)}{1+b \tau e^{-\lambda_{0} \tau}+c \lambda_{0}^{-1}\left[\lambda_{0}^{-1}\left(1-e^{-\lambda_{0} \tau}\right)-\tau e^{-\lambda_{0} \tau}\right]} \text {, for } t \in[-\tau, 0]
$$

Because of the definitions of $y$ and $z,(7)$ is equivalent to 


$$
|z(t)| \leq M(\phi)\left\{|b| \tau e^{-\lambda_{0} \tau}+|c| \lambda_{0}^{-1}\left[\lambda_{0}^{-1}\left(1-e^{-\lambda_{0} \tau}\right)-\tau e^{-\lambda_{0} \tau}\right]\right\}, \quad \forall t \geq 0 .
$$

The proof will be accomplished by proving (15).

Now, in view of (9) and (14), we have

$$
|z(t)| \leq M(\phi), \text { for } t \in[-\tau, 0]
$$

We will show that

$$
|z(t)| \leq M(\phi), \text { for all } t \in[-\tau, \infty)
$$

To this end, let us consider an arbitrary number $\varepsilon>0$. We claim that

$$
|z(t)|<M(\phi)+\varepsilon, \text { for every } t \in[-\tau, \infty) \text {. }
$$

Otherwise, by (16), there exists a $t^{*}>0$ such that

$$
|z(t)|<M(\phi)+\varepsilon, \text { for } t<t^{*} \text { and }|z(t)|=M(\phi)+\varepsilon .
$$

Then from (13), we obtain

$$
\begin{aligned}
M(\phi)+\varepsilon & =\left|z\left(t^{*}\right)\right|=\left|-b e^{-\lambda_{0} \tau} \int_{t^{*}-\tau}^{t^{*}} z(r) d r-c \int_{0}^{\tau} e^{-\lambda_{0} s}\left\{\int_{t^{*}-s}^{t^{*}} z(r) d r\right\} d s\right| \\
& \leq|b| e^{-\lambda_{0} \tau} \int_{t^{*}-\tau}^{t^{*}}|z(r)| d r+|c| \int_{0}^{\tau} e^{-\lambda_{0} s}\left\{\int_{t^{*}-s}^{t^{*}}|z(r)| d r\right\} d s \\
& \leq|b| \tau e^{-\lambda_{0} \tau}(M(\phi)+\varepsilon)+|c|(M(\phi)+\varepsilon) \int_{0}^{\tau} s e^{-\lambda_{0} s} d s \\
& =(M(\phi)+\varepsilon)\left\{|b| \tau e^{-\lambda_{0} \tau}+|c| \lambda_{0}^{-1}\left[\lambda_{0}^{-1}\left(1-e^{-\lambda_{0} \tau}\right)-\tau e^{-\lambda_{0} \tau}\right]\right\},
\end{aligned}
$$

which, in view of (5), leads to a contradiction. So, our claim is true. Since (18) holds for every $\varepsilon>0$, it follows that (17) is always satisfied. By using (17), from (13), we derive for all $t \geq 0$, 


$$
\begin{aligned}
|z(t)| & =\left|-b e^{-\lambda_{0} \tau} \int_{t-\tau}^{t} z(r) d r-c \int_{0}^{\tau} e^{-\lambda_{0} s}\left\{\int_{t-s}^{t} z(r) d r\right\} d s\right| \\
& \leq|b| e^{-\lambda_{0} \tau} \int_{t-\tau}^{t}|z(r)| d r+|c| \int_{0}^{\tau} e^{-\lambda_{0} s}\left\{\int_{t-s}^{t}|z(r)| d r\right\} d s \\
& \leq M(\phi)|b| \tau e^{-\lambda_{0} \tau}+|c| M(\phi) \int_{0}^{\tau} s e^{-\lambda_{0} s} d s \\
& \left.=M(\phi)\left\{b\left|\tau e^{-\lambda_{0} \tau}+\right| c\left|\lambda_{0}^{-1}\right| \lambda_{0}^{-1}\left(1-e^{-\lambda_{0} \tau}\right)-\tau e^{-\lambda_{0} \tau}\right]\right\},
\end{aligned}
$$

i.e., (15) holds.

The proof of our Theorem is complete.

Corollary. Assume that (4) holds and let $\lambda_{0}$ be the unique root of (3) in the interval $(\gamma, \infty)$. Then the solution $x$ of (1)-(2) satisfies

where

$$
|x(t)| \leq \Theta N(\phi) e^{\lambda_{0} t}, \text { for all } t \geq 0
$$

and

$$
\begin{gathered}
\Theta=\frac{\left(1+|b| \tau e^{-\lambda_{0} \tau}+|c| \lambda_{0}^{-1}\left[\lambda_{0}^{-1}\left(1-e^{-\lambda_{0} \tau}\right)-\tau e^{-\lambda_{0} \tau}\right]\right)^{2}}{1+b \tau e^{-\lambda_{0} \tau}+c \lambda_{0}^{-1}\left[\lambda_{0}^{-1}\left(1-e^{-\lambda_{0} \tau}\right)-\tau e^{-\lambda_{0} \tau}\right]} \\
+|b| \tau e^{-\lambda_{0} \tau}+|c| \lambda_{0}^{-1}\left[\lambda_{0}^{-1}\left(1-e^{-\lambda_{0} \tau}\right)-\tau e^{-\lambda_{0} \tau}\right]
\end{gathered}
$$

$$
N(\phi)=\max _{-\tau \leq t \leq 0}\left\{e^{-\lambda_{0} t}|\phi(t)|\right\}
$$

Moreover, the trivial solution of (1) is stable if $\lambda_{0}=0$ and it is asymptotically stable if $\lambda_{0}<0$.

Proof. First of all, we observe that, because of (5) and (6), formula (20) defines a real number $\Theta$ with $\Theta>1$.

By our theorem, (7) is satisfied, where $L(\phi)$ and $M(\phi)$ are defined by (8) and (9), respectively. From (7), it follows that

$$
\begin{aligned}
& e^{-\lambda_{0} t}|x(t)| \leq \frac{|L(\phi)|}{1+b \tau e^{-\lambda_{0} \tau}+c \lambda_{0}^{-1}\left[\lambda_{0}^{-1}\left(1-e^{-\lambda_{0} \tau}\right)-\tau e^{-\lambda_{0} \tau}\right]} \\
& \quad+M(\phi)\left\{b\left|\tau e^{-\lambda_{0} \tau}+\right| c \mid \lambda_{0}^{-1}\left[\lambda_{0}^{-1}\left(1-e^{-\lambda_{0} \tau}\right)-\tau e^{-\lambda_{0} \tau}\right]\right\}, \text { for all } t \geq 0 .
\end{aligned}
$$


But (9) gives

$$
M(\phi) \leq N(\phi)+\frac{|L(\phi)|}{1+b \tau e^{-\lambda_{0} \tau}+c \lambda_{0}^{-1}\left[\lambda_{0}^{-1}\left(1-e^{-\lambda_{0} \tau}\right)-\tau e^{-\lambda_{0} \tau}\right]} .
$$

So (22) yields

$$
\begin{aligned}
& e^{-\lambda_{0} t}|x(t)| \leq \frac{1+|b| \tau e^{-\lambda_{0} \tau}+|c| \lambda_{0}^{-1}\left[\lambda_{0}^{-1}\left(1-e^{-\lambda_{0} \tau}\right)-\tau e^{-\lambda_{0} \tau}\right]}{1+b \tau e^{-\lambda_{0} \tau}+c \lambda_{0}^{-1}\left[\lambda_{0}^{-1}\left(1-e^{-\lambda_{0} \tau}\right)-\tau e^{-\lambda_{0} \tau}\right]}|L(\phi)| \\
& +N(\phi)\left\{|b| \tau e^{-\lambda_{0} \tau}+|c| \lambda_{0}{ }^{-1}\left[\lambda_{0}{ }^{-1}\left(1-e^{-\lambda_{0} \tau}\right)-\tau e^{-\lambda_{0} \tau}\right]\right\}, \text { for every } t \geq 0 .
\end{aligned}
$$

Furthermore, from (8), we obtain

$$
|L(\phi)| \leq|\phi(0)|+|b| e^{-\lambda_{0} \tau} \int_{-\tau}^{0} e^{-\lambda_{0} \tau}|\phi(r)| d r+|c| \int_{0}^{\tau} e^{-\lambda_{0} s}\left[\int_{-s}^{0} e^{-\lambda_{0} r}|\phi(r)| d r\right] d s,
$$

which gives

$$
\left.|L(\phi)| \leq\left\{1+b\left|\tau e^{-\lambda_{0} \tau}+\right| c\left|\lambda_{0}^{-1}\right| \lambda_{0}^{-1}\left(1-e^{-\lambda_{0} \tau}\right)-\tau e^{-\lambda_{0} \tau}\right]\right\} N(\phi) .
$$

Hence, from (23), we conclude that for $t \geq 0$,

$$
\begin{gathered}
e^{-\lambda_{0} t}|x(t)| \leq \frac{\left(1+|b| \tau e^{-\lambda_{0} \tau}+|c| \lambda_{0}^{-1}\left[\lambda_{0}^{-1}\left(1-e^{-\lambda_{0} \tau}\right)-\tau e^{-\lambda_{0} \tau}\right]\right)^{2}}{1+b \tau e^{-\lambda_{0} \tau}+c \lambda_{0}^{-1}\left[\lambda_{0}^{-1}\left(1-e^{-\lambda_{0} \tau}\right)-\tau e^{-\lambda_{0} \tau}\right]} N(\phi) \\
+N(\phi)\left\{|b| \tau e^{-\lambda_{0} \tau}+|c| \lambda_{0}^{-1}\left[\lambda_{0}^{-1}\left(1-e^{-\lambda_{0} \tau}\right)-\tau e^{-\lambda_{0} \tau}\right]\right\}
\end{gathered}
$$

or

$$
e^{-\lambda_{0} t}|x(t)| \leq \Theta N(\phi)
$$

and consequently, (19) holds true.

Now, let us assume that $\lambda_{0} \leq 0$. Define

$$
\|\phi\|=\max _{-\tau \leq t \leq 0}|\phi(t)| .
$$

It follows that

$$
N(\phi) \leq\|\phi\|
$$

Thus, (19) gives 


$$
|x(t)| \leq \Theta\|\phi\| e^{\lambda_{0} t} \quad, \quad \text { for all } t \geq 0 .
$$

Since $\lambda_{0} \leq 0$, from the last inequality, it follows that

$$
|x(t)| \leq \Theta\|\phi\|, \quad \text { for every } t \geq 0 \text {. }
$$

So, by taking into account the fact that $\Theta>1$, we have

$$
|x(t)| \leq \Theta\|\phi\|, \quad \text { for every } t \in I R,
$$

which means that the trivial solution of (1) is stable. Finally, if $\lambda_{0}<0$, then (24) guarantess that

$$
\lim _{t \rightarrow \infty} x(t)=0
$$

and so the trivial solution of (1) is asymptotically stable.

\section{REFERENCES}

1. T.A. Burton, Volterra Integral and Differential Equations, Academic Press, New York, 1983.

2. C. Corduneanu, Integral Equations and Applications, Cambridge University Press, New York, 1991.

3. K. Gopalsamy, Stability ad decay rates in a class of linear integro-differential systems, Funkcial. Ekvac. 26, 251-261, 1983.

4. J.K. Hale and S.M. Verduyn Lunel, Introduction to Functional Differential Equations, Springer, Berlin, Heidelberg, New York, 1993.

5. V. Kolmanovski, A. Myshkis, Applied Theory of Functional Differential Equations, Kluver Academic, Dordrecht, 1992.

6. I.-G.E. Kordonis and Ch.G. Philos, The Behavior of solutions of linear integroDifferential equations with unbounded delay, Comp. and Math. with Appl. 38, 45-50, 1999.

7. T. Koto, Stability of Runge-Kutta methods for delay integro-differential equations, J. Of Comp. and Appl. Math. 145, 483-492, 2002.

8. Y. Kuang, Delay Differential Equations with Applications in Population Dynamics, Academic Press, San Diego, 1993.

9. Ch.G. Philos and I.K. Purnaras, Periodic first order linear neutral delay differential equations, Appl. Math. and Comp. 117, 203-222, 2001.

10. V. Volterra, Sur la théorie mathématique des phénoménes héréditaires, J. Math. Pures Appl. 7(9), 249-298, 1928. 